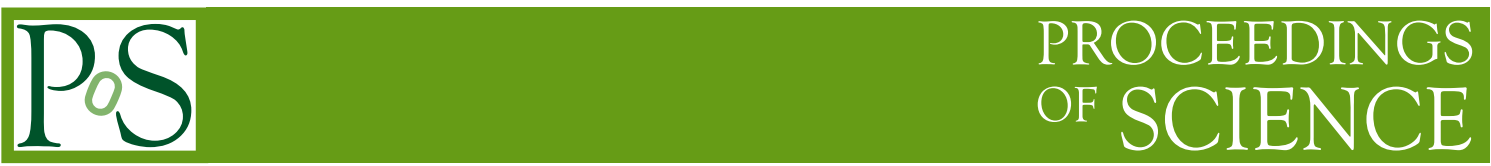

\title{
High-energy neutrinos from blazar flares
}

\author{
Foteini Oikonomou* \\ European Southern Observatory, Garching bei München, Germany \\ E-mail: foikonomeeso.org
}

The IceCube Collaboration recently reported the observation of a $\gtrsim 290 \mathrm{TeV}$ muon neutrino, IceCube-170922A, coincident with a $\sim 6$-month-long $\gamma$-ray flare of the blazar TXS 0506+056. The neutrino detection prompted electromagnetic follow-up of the event, and the blazar flare was detected by several instruments, including MAGIC at energies exceeding $>100 \mathrm{GeV}$. The correlation of the neutrino with the flare of TXS 0506+056 is inconsistent with arising by chance at the $3 \sigma$ level. An archival search revealed $13 \pm 5$ further high-energy neutrinos in the direction of TXS 0506+056 during a 6-month period in 2014-2015. These events were not accompanied by a $\gamma$-ray flare. Such an accumulation of events is inconsistent with arising from a background fluctuation at the $3.5 \sigma$ level. Here, recent results on neutrino emission expectations, aimed at interpreting, and motivated by these observations are summarised. The topics briefly reviewed include models of neutrino production in TXS 0506+056 during the $2017 \gamma$-ray flare, during the 2014-2015 period, and expectations of neutrino emission from blazar flares from other sources that occurred in the period 2008-2018 in declinations favourable for detection with IceCube.

7th Annual Conference on High Energy Astrophysics in Southern Africa ***

28 - 30 August 2019

Swakopmund, Namibia

${ }^{*}$ Speaker. 


\section{Introduction}

Active galactic nuclei (AGN) hosting a strong relativistic jet, are the most powerful persistent astrophysical sources in the Universe. Blazars are jetted AGN whose jets are oriented at a small angle with respect to the line of sight [1]. Blazars form the majority of known extragalactic $\gamma$-ray sources [2]; as such they have long been discussed as some of the most likely sources of highenergy neutrinos and cosmic-rays (see $[3,4,5,6,7,8,9,10,11,12,13,14,15]$, and references therein).

The IceCube South Pole Neutrino Observatory ${ }^{1}$ first reported the observation of high-energy astrophysical neutrinos $[16,17,18]$ a few years ago. Most recently, the neutrino flux collected over 6 years with deposited energy up to about $2 \mathrm{PeV}$ was reported in [19], bringing the number of high-energy starting neutrino events detected to 82 and strengthening the significance $(>6.5 \sigma)$ of the observation that they are incompatible with being of purely terrestrial origin.

Observations of the diffuse, all-sky, neutrino spectrum by IceCube have resulted in constraints on the time-averaged emission from $\gamma$-ray bright blazars as sources of high-energy neutrinos from analyses of the observed diffuse neutrino flux [20, 19], and stacking analyses [21, 22], down to $\lesssim 10-30 \%$ of the diffuse astrophysical flux observed by IceCube at $\lesssim 100 \mathrm{TeV}$. Thus, blazars are inconsistent with being the dominant sources of the astrophysical neutrino flux detected by IceCube. Constraints on blazars as dominant sources of the diffuse neutrino intensity observed by IceCube are additionally imposed by the lack of multiplets in the IceCube data [23, 24, 22, 25, 26], which limits the blazar contribution to the diffuse IceCube flux [27] in a complimentary way.

Despite these limits, the brightest neutrino sources could still be blazars, considering how bright they are individually. Blazar flaring periods are ideal for the detection of high-energy neutrinos, both observationally and theoretically. Observationally, the short, well-defined, time duration of a flare means a very reduced background rate. Theoretically, in many models of neutrino emission, it is natural for neutrino production to be strongly enhanced during flares because it is typically expected that the proton injection increases while at the same time the target photon field is in an enhanced state (see e.g. [9, 28, 29, 23, 30, 15]). It was shown in [26] that even though blazars emit a small fraction of their $\gamma$-ray luminosity during flares, e.g. [31, 32], the distribution of flaring states of several studied Fermi blazars is consistent with neutrino production during flares being dominant in canonical models of neutrino emission.

The IceCube Collaboration has recently reported the observation of a $\gtrsim 290 \mathrm{TeV}$ muon neutrino, IceCube-170922A, coincident with a 6-month-long $\gamma$-ray flare of the blazar TXS 0506+056 [33] at redshift $z=0.3365$ [34]. The neutrino detection prompted electromagnetic follow-up of the event, and the blazar flare was detected by several instruments, including MAGIC at energies exceeding $>100 \mathrm{GeV}$. The correlation of the neutrino with the flare of TXS 0506+056 is inconsistent with arising by chance at the $\sim 3 \sigma$ level. An archival search revealed $13 \pm 5$ further, high-energy neutrinos in the direction of TXS 0506+056 during a 6-month period in 2014-2015 [35]. These events were not accompanied by a $\gamma$-ray flare. Such an accumulation of events is inconsistent with arising from a background fluctuation at the $3.5 \sigma$ level.

In this proceeding, the results of studies of the neutrino emission from TXS 0506+056 and other blazar flares are summarised from a theoretical perspective. Specifically the questions ad-

\footnotetext{
${ }^{1}$ http://icecube.wisc.edu
} 
dressed include, what is the theoretically maximal allowed neutrino emission of TXS 0506+056 during its recent flares in canonical blazar emission models, what is the maximal emission expected in general in blazar models in the case of rare events or exceptional conditions, and finally what is the expected neutrino emission from other blazar flares that occurred in the field of view of IceCube and might have therefore also been detectable?

\section{Neutrino emission in TXS $0506+056$}

A plausible and widely considered means of production of high-energy neutrinos in blazar jets is the interactions of protons in the acceleration region (commonly referred to as "blazar zone" or "blob") of the jet, with photons from the internally produced synchrotron radiation in the blob, or with radiation fields external to the jet, which include the Broad Line Region (BLR) and Dust Torus. We shall refer to the photohadronic interactions as $p \gamma$ interactions.

Based on their optical spectra, blazars are divided into two main sub-classes, namely BL Lacertae objects (BL Lacs) and Flat Spectrum Radio Quasars (FSRQs). FSRQs, which possess more powerful jets, display broad, strong emission lines, which reveal the presence of an efficient accretion disk able to photo-ionise molecular clouds. BL Lacs exhibit at most weak emission lines, or in many cases featureless optical spectra and have weaker jets. Neutrino production is expected to be more efficient in FSRQs than in BL Lac objects due to the higher-powers and existence of external photon fields [36]. In [37] we presented evidence that TXS 0506+056 is, despite its generally accepted classification, intrinsically an FSRQ. This implies the presence of the intense radiation field of the BLR and should allow for enhanced neutrino production if the neutrino emitting region is at a small distance from the base of the jet, i.e. within the BLR.

In very general terms, the expected neutrino flux from an astrophysical source from $p \gamma$ interactions depends on the high-energy proton luminosity of the source, the efficiency of $p \gamma$ interactions which depends on the energy density of radiation fields in in the source environment, and the escape timescale and all other energy loss-timescales for the protons in the source environment. Below some basic arguments are outlined.

The optical depth for protons to $p \gamma$ interactions, $f_{p \gamma}$, is given by, $\left.f_{p \gamma}\left(\varepsilon_{p}\right) \approx \hat{\sigma}_{p \gamma} r_{b}^{\prime} n_{\mathcal{\varepsilon}_{t}^{\prime}} \varepsilon_{t}^{\prime}\right|_{\varepsilon_{t}^{\prime}=0.5 \overline{\varepsilon_{\Delta}} m_{p} c^{2} / \varepsilon_{p}^{\prime}}$, e.g. [38]. Here, the effective $p \gamma$ cross-section at threshold, $\hat{\sigma}_{p \gamma} \sim 0.7 \times 10^{-28} \mathrm{~cm}^{2}, r_{b}^{\prime}$ is the radius of the emitting blob, $n_{\varepsilon_{t}^{\prime}}$ is the density of target photons of energy $\varepsilon_{t}^{\prime}$ and $\varepsilon_{p}^{\prime}$ the proton energy all in the frame comoving with the blob. Further, $m_{p}$ is the proton mass, $m_{\pi}$ the pion mass and $\overline{\varepsilon_{\Delta}}$ the energy required to produce the $\Delta$ resonance.

The same photons are the target photons for $\gamma \gamma$ interactions. The relevant optical depth is related to $f_{p \gamma}$, via, $\left.\tau_{\gamma \gamma}\left(\varepsilon_{\gamma}\right) \approx r_{\mathrm{b}}^{\prime} \sigma_{\gamma \gamma} \varepsilon_{t}^{\prime} n_{\varepsilon_{t}^{\prime}}\right|_{\varepsilon_{t}^{\prime}=m_{e}^{2} c^{4} / \varepsilon_{\gamma}^{\prime}}$, with $\sigma_{\gamma \gamma} \sim 0.1 \sigma_{\mathrm{T}} \sim 10^{-25} \mathrm{~cm}^{2}$ the Thompson cross-section. The ratio of optical depths from the two processes is then,

$$
f_{p \gamma}\left(\varepsilon_{p}\right) \approx \frac{\sigma_{p \gamma}}{\sigma_{\gamma \gamma}} \tau_{\gamma \gamma}\left(\varepsilon_{\gamma}\right) \approx 10^{-3} \tau_{\gamma \gamma}\left(\varepsilon_{\gamma}\right)
$$

at energy,

$$
\varepsilon_{\gamma} \sim 15 \mathrm{GeV}\left(\frac{\varepsilon_{p}}{6 \mathrm{PeV}}\right) \sim 15 \mathrm{GeV}\left(\frac{\varepsilon_{v}}{300 \mathrm{TeV}}\right) .
$$

The above relations reveal that in order to have increased neutrino production in a source we need to have increased neutrino production efficiency, $f_{p \gamma}$, which is generally achieved when there 
is a dense radiation field, or a very extended one. In FSRQs the BLR provides a radiation field with large energy density. In BL Lac objects the BLR is absent, but there is evidence of a stratified jet, with a slower outer region which may be very extended, referred to as the "spine-sheath" model [40], as well as the radiation field of the inefficient accretion flow [41]. Both these fields, if present, enhance $f_{p \gamma}$. On the other hand, equations 2.1 and 2.2 tell us that in $\gamma$-ray bright sources, we cannot increase $f_{p \gamma}$ arbitrarily, because if we do so the optical depth for $\gamma$-rays to escape the source would be too high.

The observation of $>10-100 \mathrm{GeV}$ photons from TXS 0506+056 during the 2017 flare, implies that the optical depth for photons to $\gamma \gamma$ interactions on low-energy photons, $\tau_{\gamma \gamma}\left(\varepsilon_{\gamma}=10-100 \mathrm{GeV}\right)<1$ and thus a limit to $f_{p \gamma}\left(\varepsilon_{p}=6 \mathrm{PeV}\right)<10^{-3}$. The limit to $f_{p \gamma}$ thus constrains the maximum neutrino luminosity, $L_{v}$, if the neutrino and radiative power are cospatialy produced.

Of the energy protons lose in $p \gamma$ interactions, $3 / 8$ ths go to neutrino production. For proton luminosity, $\varepsilon_{p} L_{\varepsilon_{p}}$, the differential neutrino luminosity is then given by,

$$
\varepsilon_{v} L_{\varepsilon_{v}} \approx \frac{3}{8} f_{p \gamma}\left(\varepsilon_{p} L_{\varepsilon_{p}}\right) \simeq 1.2 \times 10^{45} \mathrm{erg} \mathrm{s}^{-1} \frac{f_{p \gamma}}{10^{-4}}\left(\frac{\varepsilon_{p} L_{\varepsilon_{p}}}{10^{49.5} \mathrm{erg} \mathrm{s}^{-1}}\right) .
$$

Alternatively, if $f_{p \gamma}$ cannot increase further, the higher the proton luminosity of the source, the higher the neutrino luminosity. However, additionally, the protons undergo Bethe-Heitler interactions [42, 43], with effective optical depth, $\left.f_{B H}\left(\varepsilon_{p}\right) \approx \hat{\sigma}_{\mathrm{BH}} r_{b}^{\prime} n_{\mathcal{\varepsilon}_{t}^{\prime}} \varepsilon_{t}^{\prime}\right|_{\mathcal{\varepsilon}_{t}^{\prime}=m_{p} c^{2} \bar{\varepsilon}_{\mathrm{BH}} / 2 \varepsilon_{p}^{\prime}}=g[\beta] f_{p \gamma}\left[\varepsilon_{p}\right]$, where, $\hat{\sigma}_{\mathrm{BH}} \sim 0.8 \times 10^{-30} \mathrm{~cm}^{2}, g[\beta] \sim 0.011(30)^{\beta-1}$, and $\bar{\varepsilon}_{\mathrm{BH}} \sim 10\left(2 m_{e} c^{2}\right) \sim 10 \mathrm{MeV}$. Thus, a further limit is imposed on the maximum neutrino luminosity and therefore indirectly on the maximum proton luminosity from the requirement that the synchrotron cascade flux produced by electron-positron pairs, injected by Bethe-Heitler interactions, should not exceed the observed Xray flux,

$$
\left.\varepsilon_{\gamma} L_{\varepsilon_{\gamma}}^{X}\right|_{\varepsilon_{\mathrm{syn}} \mathrm{BH}} \approx \frac{1}{2\left(1+Y_{\mathrm{IC}}\right)} g[\beta] f_{p \gamma} \varepsilon_{p} L_{p} \approx \frac{4}{3\left(1+Y_{\mathrm{IC}}\right)} g[\beta] \varepsilon_{v} L_{\varepsilon_{v}}
$$

where, $\varepsilon_{\mathrm{syn}}^{\mathrm{BH}} \approx 6 \mathrm{keV} B_{0.5 \mathrm{G}}^{\prime}\left(\varepsilon_{p} / 6 \mathrm{PeV}\right)^{2}(20 / \delta)$, with $\delta$ the Doppler factor of the relativistic motion of the emitting region, $B^{\prime}$ the magnetic field strength as measured by a comoving observer, and $Y_{\mathrm{IC}}$ the Compton dominance parameter which is at most 1 for TXS 0506+056 [39].

For the flaring spectrum of TXS 0506+056 in 2017 from the analysis of [39] this corresponds to $\varepsilon_{\gamma} L_{\varepsilon_{\gamma}}^{X} \leq 3 \times 10^{44} \mathrm{erg} / \mathrm{s}$. This imposes a constraint on the maximum muon neutrino luminosity at the level of, $\varepsilon_{v} L_{\varepsilon_{v_{\mu}}}^{0.1-1 \mathrm{PeV}} \lesssim \varepsilon_{\gamma} L_{\varepsilon_{\gamma}}^{X} / 3 \sim 10^{44} \mathrm{erg} \mathrm{s}^{-1}$, where the factor of 3 accounts for going from all-flavour to single-flavour neutrino luminosity, assuming $Y_{\mathrm{IC}}=1$ which gives the most optimistic estimate for the maximum neutrino luminosity of TXS 0506+056.

In addition, a synchrotron cascade flux component from electron-positron pairs injected from photo-meson production and electron-positron pair production from hadronic $\gamma$-rays is unavoidable in the single-zone model. The minimum cascade flux from these processes is,

$$
\left.\varepsilon_{\gamma} L_{\varepsilon_{\gamma}}\right|_{\varepsilon_{\mathrm{syn}}^{p \gamma}} \approx \frac{5}{8} \frac{1}{2\left(1+Y_{\mathrm{IC}}\right)} f_{p \gamma}\left(\varepsilon_{p} L_{\varepsilon_{p}}\right) \approx \frac{5}{6\left(1+Y_{\mathrm{IC}}\right)} \varepsilon_{v} L_{\varepsilon_{v}}
$$

where $\left.\varepsilon_{\mathrm{syn}}^{p \gamma} \simeq 60 \mathrm{MeV}\left(B^{\prime} / 0.3 \mathrm{G}\right)\right)\left(\varepsilon_{p} / 6 \mathrm{PeV}\right)^{2}(20 / \delta)$. For the 2017 flare of TXS 0506+056, the $\mathrm{X}$-ray cascade constraint of eq. 2.4 is stronger. 
The conclusion from the above considerations is that if neutrino emission occurs in the same part of the jet of TXS 0506+056 as the photon emission, at most $N_{v_{\mu}+\overline{v_{\mu}}} \sim 0.01$ can be expected in the IceCube EHE channel during the six month 2017 flare of TXS 0506+056 above $100 \mathrm{TeV}$. Cospatial production is in general expected if the neutrino emission is correlated with the $\gamma$-ray flare. In this sense, consistent conclusions were reached by the majority of studies on the 2017 flare of TXS 0506+056 [44, 39, 45, 46].

It was pointed out by $[33,47]$ that in an ensemble of faint sources with summed expectation of order 1 , one might observe a neutrino even if the individual expectation value is $\ll 1$. Hence the observation of a single neutrino only in 2017 gives an upper limit of the neutrino flux of TXS 0506+056, which is consistent with the expected neutrino counts after accounting for the existence of such a source ensemble.

Other works focussed exclusively on the 2014-15 flare. In Rodriguez et al [48] it was shown that in canonical blazar models, even if one invokes more than one emitting zones, it is not possible to produce more than $\sim 5$ muon neutrinos over the duration of the 2014-15 flare without overproducing the radiative cascade. This result agrees with the result of [49] who modelled the 2014-15 spectrum of TXS 0506+056 and [50] who performed multi-epoch modelling of the source and who concluded that if the $13 \pm 5$ neutrinos were produced by TXS $0506+056$ they were produced in a region other than the blazar zone where the bulk of the observed radiation was produced.

If the conditions in the environment of TXS 0506+056 are not representative of conventional blazar models, or the neutrino emission is associated with a rare event, then some additional possibilities for more intense neutrino production exist.

In [51] it was shown that the neutrino emission of TXS 0506+056 during its 2017 flare can be interpreted as inelastic hadronuclear interactions between the accelerated cosmic-ray protons in the relativistic jet and the dense gas clouds of the BLR. In this scenario the required proton power in the jet is much smaller than in conventional $p \gamma$ interaction models. However, in this scenario the neutrino emission is not necessarily correlated with the $\gamma$-ray flare.

In [52] it was shown that if TXS 0506+056 has two distinct emitting regions, namely, one compact emitting region within the BLR responsible for the neutrino emission and some of the $\gamma$-ray emission, and another beyond the BLR responsible for the synchrotron emission, then it is possible to produce larger amounts of neutrinos than standard $p \gamma$ models where all the emission is cospatially produced. If such a mechanism is in operation, i.e. if such an emitting region exists inside the BLR it can at most occur in $10 \%$ of blazars or less than $5 \%$ of the time, otherwise it would over-produce the observed astrophysical neutrino flux.

In [53] it was proposed that the 2014-2015 neutrino outburst of TXS 0506+056 originates in the interaction of the relativistic jet and a dense gas cloud which may be formed via the tidally disrupted envelope of a red giant being blown by the impact of the jet.

In [54] an alternate model was proposed in which copious amounts of neutrinos are produced if large amounts of cosmic-ray nuclei, and specifically Helium, are accelerated in the blazar zone. In this case, if neutrons, produced in the interactions of the cosmic-ray nuclei, travel down the jet producing neutrinos while protons remain magnetically confined, significantly larger amounts of neutrinos are produced than in $p \gamma$ models, consistent with the IceCube neutrino observations of the source, in 2014-15 and 2017 without being in conflict with the observed photon spectral energy 
distribution. However the required radiation density of external photon fields in this model so as to reproduce the IceCube 2014-15 observations is atypically large.

In [55], evidence was presented, based on reanalysis of ten years of MOJAVE data, that TXS 0506+056 has a very curved jet, or possibly even a second jet. Such a special geometry could allow for significantly enhanced neutrino emission with respect to typical blazars, if the jet self-interacts or if two jets interact.

\section{Other flares}

In [56] we collected publicly available data for recorded flaring episodes of 12 Fermi bright BL Lacs, for which simultaneous (or semi-simultaneous) observations exist, with sufficient spectral coverage as to infer the main characteristics of the spectral energy distribution during the flare with reasonable confidence (for example the peak synchrotron frequency and peak synchrotron flux).

We focussed on objects classified as BL Lacs, in which the bulk of the emission can in general be attributed to synchrotron and synchrotron self-Compton (SSC) processes. Even though generally BL Lac objects are expected to produce lower neutrino fluxes than FSRQs, our sample here contains several FSRQs which, like TXS 0506+056 have been misclassified as BL Lacs and are very powerful sources. The details of all the flares in our sample are given in Tab. 1.

\subsection{Model assumptions}

One of the models we assumed to simulate the source environment of the studied sources was inspired by the most optimistic model of [39], LMPL2b, for the September 2017 flare of TXS 0506+056 (see also their Tab. 7), if IceCube-170922A was indeed produced by TXS 0506+056. The assumed proton luminosity is higher than the $\gamma$-ray luminosity, by a factor $\xi$. By default we used $\xi_{\mathrm{cr}}=1540$ which implies that the proton luminosity of these sources was in the range $0.2-30$ times the Eddington luminosity during the flaring episode.

The maximum proton energy is low, of order $\mathrm{PeV}$, and as a result the neutrino spectrum typically peaks at $\sim$ sub-PeV energies. We consider the interactions of protons with internally produced synchrotron radiation in the blob, and additionally we consider the interactions of protons with an external photon field inspired by the spine-sheath model [40], with spectrum as in the LMPL2b model of [39]. Generally, the sheath photon field cannot be much higher than the values assumed in this work, in one-zone models [57].

\subsection{Results}

Figure 1 shows the modelled spectral energy distributions of all the studied flaring sources, and the expected instantaneous neutrino spectra within our model assumptions. Figure 2 summarises the expected neutrino counts, above $100 \mathrm{TeV}$ where the atmospheric background is low, in our optimistic model, with IceCube (red bars) and with future, planned and proposed neutrino facilities (blue bars) stacking ten years of Fermi flares for each of the studied sources. Neutrinos are not above the detection threshold for any of the studied sources which include TXS 0506+056 individually with our model while IceCube is in operation alone. To forecast the expected neutrino counts in future facilities we assumed that the upgraded IceCube-Gen2 experiment will have effective area six-times larger than IceCube and that additionally three neutrino telescopes with 

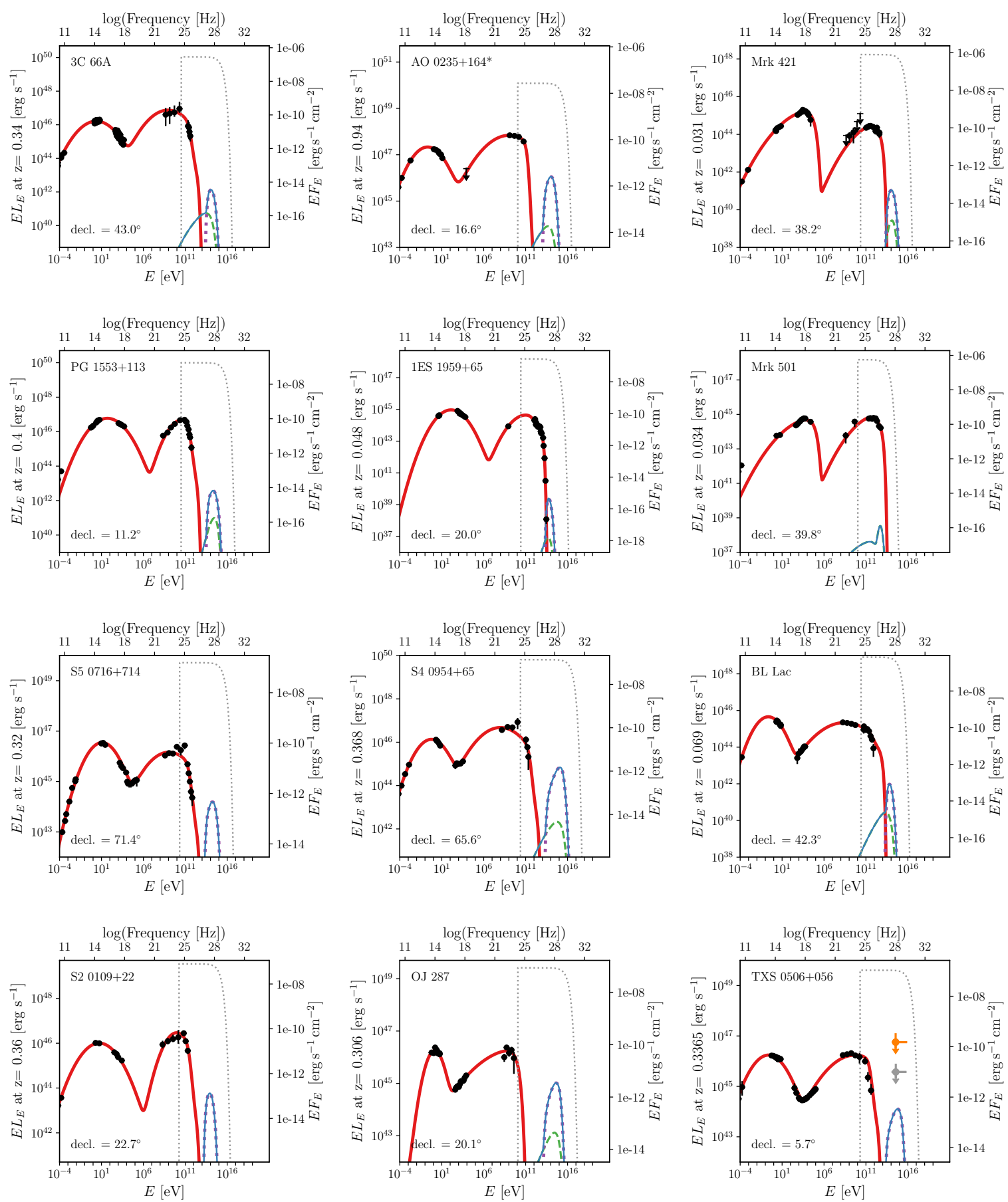

Figure 1: Broadband spectral energy distribution (red), injected proton flux (black dotted lines), and expected instantaneous all-flavour neutrino flux (blue) for each for the flares in our sample in the observer frame. Green dashed lines give the neutrino emission produced in interactions of protons with photons the blob. For some of the flares these are very suppressed and not seen in the plots. Purple dotted lines give the neutrino flux produced in interactions of protons with photons of the stationary external field. Blue solid lines give the total neutrino emission. ${ }^{\star}$ The star marks that for AO $0235+164$ we have used baryon loading ten times lower than all other sources, $\xi_{\mathrm{cr}}=150$. 


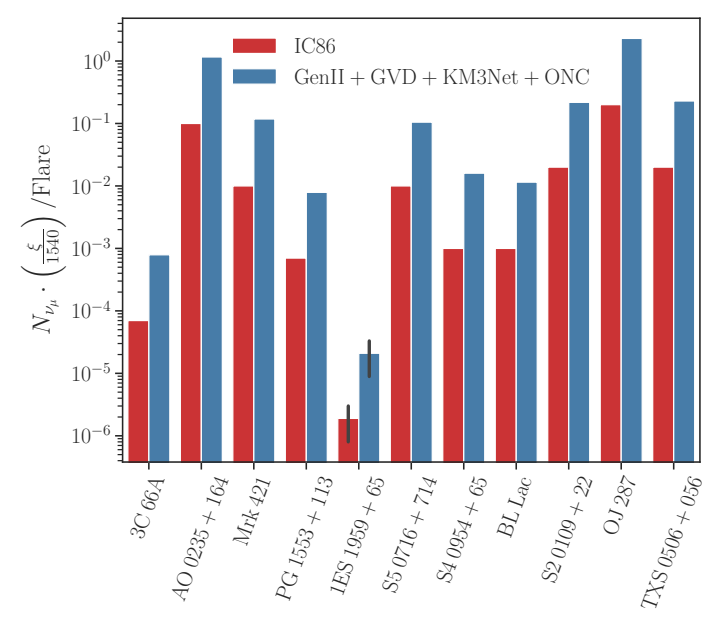

Figure 2: Predicted neutrino counts for each of the sources in our sample. The red bars give the expected number of muon neutrinos as seen in IceCube, in the IC86 configuration, and comparison to the expected number of neutrinos for identical flares if IceCube-Gen2 were in operation, as well as three additional IceCube-sized detectors in the Northern hemisphere. For 1ES 1959+650 the error bars are statistical and derive from the modelling of two separate flares. ${ }^{\star}$ For AO $0235+164$ we have used baryon loading ten times lower than all other sources, $\xi_{\mathrm{cr}}=150$.

effective area identical to that of IceCube will be operating in the Northern hemisphere (see [56] for details of the modelled facilities). In this configuration, the two brightest sources in our sample, $\mathrm{AO} 0235+164$, and $\mathrm{OJ} 287$ would produce in total $\sim 3$ muon+anti-muon neutrinos above $100 \mathrm{TeV}$ or otherwise, constrain the proton luminosity of their jets to be lower than assumed in our optimistic model.

\section{Summary}

This proceeding summarises recent results on blazars, and blazar flares as sources of the diffuse neutrino flux seen with IceCube, and as neutrino point sources, respectively. The constraints on neutrino production in TXS 0506+056 during its 2017 and 2014-15 flares, in canonical blazar jets, for neutrino emission cospatial with the radiative output of the jet were outlined, and expected neutrino counts from other blazar flares were presented. The continued operation of IceCube, the advent of KM3NeT [58], IceCube-Gen2 and other future neutrino telescopes in concert with multimessenger observations, will continue to shed light to the question of the origin of high-energy neutrinos, and the proton content of blazar jets.

\section{References}

[1] C. M. Urry and P. Padovani, Publ. Astron. Soc. Pac. 107 (1995) 803 [astro-ph/ 9506063 ].

[2] Fermi-LAT collaboration, 1902.10045.

[3] K. Mannheim, Astropart. Phys. 3 (1995) 295.

[4] F. Halzen and E. Zas, ApJ 488 (1997) 669. 


\begin{tabular}{ccccc}
\hline Source & $\mathrm{z}$ & decl. & UT & $\Delta T$ \\
\hline 3C 66A & 0.34 & +43.04 & Oct 2008 & 14 \\
AO 0235+164 & 0.94 & +16.62 & Oct 2008 & 84 \\
Mrk 421 & 0.031 & +38.21 & Mar 2010 & 13 \\
PG 1553+113 & $\sim 0.4$ & +11.19 & Apr 2012 & 30 \\
1ES 1959+650a & 0.048 & +20.00 & May 2012 & 46 \\
Mrk 501 & 0.033 & +39.76 & Jun 2012 & 21 \\
S5 0716+714 & $<0.32$ & +71.37 & Jan 2015 & 14 \\
S4 0954+65 & $\geq 0.45$ & +65.57 & Feb 2015 & 28 \\
BL Lac & 0.07 & +42.28 & Jun 2015 & 7 \\
S2 0109+22 & 0.36 & +22.74 & Jul 2015 & 21 \\
1ES 1959+650b & 0.048 & +20.00 & Sep 2015 & 84 \\
OJ 287 & 0.306 & +20.11 & Dec 2015 & 7 \\
TXS 0506+056 & 0.3365 & +5.70 & Sep 2017 & 175 \\
\hline
\end{tabular}

Table 1: The list of flares studied in this work. For each flare the table lists the redshift (or assumed redshift) of the source, declination, the month of onset of the flare, and the flare duration, which we determined by analysis of the $>800 \mathrm{MeV}$ FAVA [59] lightcurves. The references for the observational campaigns are listed in [56].

[5] A. Atoyan and C. D. Dermer, Phys.Rev.Lett. 87 (2001) 221102 [astro-ph/ 0108053 ].

[6] A. Mücke, R. J. Protheroe et al., Astropart. Phys. 18 (2003) 593.

[7] K. Murase, C. D. Dermer et al., ApJ 749 (2012) 63 [1107. 5576].

[8] C. D. Dermer, K. Murase et al., ApJ 755 (2012) 147 [1203. 6544 ].

[9] K. Murase, Y. Inoue et al., Phys. Rev. D 90 (2014) 023007 [1403. 4089 ].

[10] P. Padovani and E. Resconi, MNRAS 443 (2014) 474 [1 406 . 0376].

[11] C. D. Dermer, K. Murase et al., JHEAp 3-4 (2014) 29 [1406.2633].

[12] M. Petropoulou, S. Dimitrakoudis et al., MNRAS 448 (2015) 2412 [1501 . 07115].

[13] P. Padovani, E. Resconi et al., MNRAS 457 (2016) 3582 [1601. 06550].

[14] S. Gao, M. Pohl et al., ApJ 843 (2017) 109 [1610 . 05306].

[15] X. Rodrigues, A. Fedynitch et al., ApJ 854 (2018) 54 [1711.02091].

[16] IceCube Collaboration, Phys. Rev. Lett. 111 (2013) 021103.

[17] IceCube Collaboration, Science 342 (2013) .

[18] IceCube Collaboration, Phys. Rev. Lett. 113 (2014) 101101.

[19] IceCube Collaboration in Proceedings of ICRC 2017, 2017, 1710.01191.

[20] IceCube Collaboration, Phys. Rev. Lett. 117 (2016) 241101 [1607 . 05886].

[21] IceCube Collaboration, ApJ 835 (2017) 45.

[22] IceCube Collaboration in Proceedings of ICRC 2017, 2017, 1710.01179.

[23] K. Murase and E. Waxman, Phys. Rev. D 94 (2016) 103006 [1607 . 01601]. 
[24] IceCube Collaboration, Astropart. Phys. 66 (2015) 39 [1408 . 0634].

[25] A. Neronov and D. V. Semikoz, 1811.06356.

[26] K. Murase, F. Oikonomou et al., ApJ 865 (2018) 124 [1807. 04748].

[27] C. Yuan, K. Murase et al., 1904.06371.

[28] F. Tavecchio, G. Ghisellini et al., Astrophys.J. 793 (2014) L18.

[29] M. Petropoulou, S. Coenders et al., Astropart. Phys. 80 (2016) 115 [1603. 06954 ].

[30] C. Righi, F. Tavecchio et al., A\&A 598 (2017) A36 [1607. 08061 ].

[31] Fermi-LAT Collaboration, ApJ 722 (2010) 520 [1004.0348].

[32] Fermi-LAT Collaboration, ApJ 743 (2011) 171 [1108 . 1420].

[33] IceCube Collaboration, Fermi-LAT et al., Science 361 (2018) eaat1378 [1807.08816].

[34] S. Paiano, R. Falomo et al., ApJ 854 (2018) L32 [1802 . 01939].

[35] ICECUBE collaboration, Science 361 (2018) 147 [1807.08794].

[36] A. M. Atoyan and C. D. Dermer, ApJ 586 (2003) 79 [astro-ph / 0209231 ].

[37] P. Padovani, F. Oikonomou et al., MNRAS 484 (2019) L104 [1901. 06998].

[38] K. Murase, D. Guetta et al., Phys. Rev. Lett. 116 (2016) 071101 [1509. 00805 ].

[39] A. Keivani, K. Murase et al., ApJ 864 (2018) 84 [1807. 04537 ].

[40] G. Ghisellini, F. Tavecchio et al., A\&A 432 (2005) 401 [astro-ph/ 0406093 ].

[41] C. Righi, F. Tavecchio et al., MNRAS 483 (2019) L127 [1807 . 10506].

[42] K. Murase and J. F. Beacom, Phys. Rev. D82 (2010) 043008 [1 002 . 3980].

[43] M. Petropoulou and A. Mastichiadis, MNRAS 447 (2015) 36 [1411. 1908].

[44] S. Gao, A. Fedynitch et al., Nature Astronomy (2018) 154 [1807. 04275].

[45] M. Cerruti, A. Zech et al., MNRAS 483 (2019) L12 [1807 . 04335].

[46] MAGIC collaboration, ApJ 863 (2018) L10 [1807. 04300].

[47] N. L. Strotjohann, M. Kowalski et al., A\&A 622 (2019) L9 [1809 . 06865].

[48] X. Rodrigues, S. Gao et al., ApJ 874 (2019) L29 [1812 . 05939].

[49] A. Reimer, M. Böttcher et al., ApJ 881 (2019) 46 [1812 . 05654].

[50] M. Petropoulou, K. Murase et al., 1911.04010.

[51] R.-Y. Liu, K. Wang et al., Phys. Rev. D99 (2019) 063008 [1807. 05113].

[52] R. Xue, R.-Y. Liu et al., ApJ 886 (2019) 23.

[53] K. Wang, R.-Y. Liu et al., 1809.00601.

[54] B. T. Zhang, M. Petropoulou et al., 1910.11464.

[55] Britzen, S., Fendt, C. et al., A\&A 630 (2019) A103.

[56] F. Oikonomou, K. Murase et al., Mon. Not. Roy. Astron. Soc. 489 (2019) 4347 [1 906.05302 ].

[57] F. Tavecchio, F. Oikonomou et al., Mon. Not. Roy. Astron. Soc. 488 (2019) 4023 [190 6 . 02521].

[58] KM3NET collaboration, KM3NeT Collaboration, KM3NeT: Technical Design Report. 2009.

[59] Fermi-LAT Collaboration, ApJ 846 (2017) 34 [1612 . 03165]. 